\title{
Rediscovery of the transcerebellar approach: improving the risk-benefit ratio in robot-assisted brainstem biopsies
}

\author{
Kathrin Machetanz, MD, ${ }^{1,2}$ Florian Grimm, MD, ${ }^{1,2}$ Sophie Wang, MD, ${ }^{1}$ Martin U. Schuhmann, MD,1 \\ Marcos Tatagiba, MD, ${ }^{1}$ Alireza Gharabaghi, MD, ${ }^{1,2}$ and Georgios Naros, MD ${ }^{1,2}$

\begin{abstract}
${ }^{1}$ Neurosurgical Clinic, Department of Neurosurgery and Neurotechnology, Eberhard Karls University, Tuebingen; ${ }^{2}$ Institute for Neuromodulation and Neurotechnology, Department of Neurosurgery and Neurotechnology, Eberhard Karls University, Tuebingen, Germany
\end{abstract}

\begin{abstract}
OBJECTIVE Conventional frame-based stereotaxy through a transfrontal approach (TFA) is the gold standard in brainstem biopsies. Because of the high surgical morbidity and limited impact on therapy, brainstem biopsies are controversial. The introduction of robot-assisted stereotaxy potentially improves the risk-benefit ratio by simplifying a transcerebellar approach (TCA). The aim of this single-center cohort study was to evaluate the risk-benefit ratio of transcerebellar brainstem biopsies performed by 2 different robotic systems. In addition to standard quality indicators, a special focus was set on trajectory selection for reducing surgical morbidity.

METHODS This study included 25 pediatric $(n=7)$ and adult $(n=18)$ patients who underwent 26 robot-assisted biopsies via a TCA. The diagnostic yield, complication rate, trajectory characteristics (i.e., length, anatomical entry, and target-point location), and skin-to-skin (STS) time were evaluated. Transcerebellar and hypothetical transfrontal trajectories were reconstructed and transferred into a common MR space for further comparison with anatomical atlases.
\end{abstract}

RESULTS Robot-assisted, transcerebellar biopsies demonstrated a high diagnostic yield (96.2\%) while exerting no surgical mortality and no permanent morbidity in both pediatric and adult patients. Only $3.8 \%$ of cases involved a transient neurological deterioration. Transcerebellar trajectories had a length of $48.4 \pm 7.3 \mathrm{~mm}$ using a wide stereotactic corridor via crus I or II of the cerebellum and the middle cerebellar peduncle. The mean STS time was $49.5 \pm 23.7$ minutes and differed significantly between the robotic systems $(p=0.017)$. The TFA was characterized by longer trajectories $(107.4 \pm$ $11.8 \mathrm{~mm}, p<0.001)$ and affected multiple eloquent structures. Transfrontal target points were located significantly more medial $(-3.4 \pm 7.2 \mathrm{~mm}, \mathrm{p}=0.042)$ and anterior $(-3.9 \pm 8.4 \mathrm{~mm}, \mathrm{p}=0.048)$ in comparison with the transcerebellar trajectories.

CONCLUSIONS Robot-assisted, transcerebellar stereotaxy can improve the risk-benefit ratio of brainstem biopsies by avoiding the restrictions of a TFA and conventional frame-based stereotaxy. Profound registration and anatomicalfunctional trajectory selection were essential to reduce mortality and morbidity.

https://thejns.org/doi/abs/10.3171/2021.10.FOCUS21359

KEYWORDS brainstem tumors; cerebellar anatomy; robot-assisted biopsies; transcerebellar approach; transfrontal approach

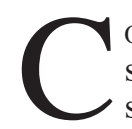

ONVENTIONAL frame-based stereotaxy is the gold standard for precise intracranial biopsies. ${ }^{1,2}$ Considering the low morbidity rate (approximately $0 \%-8.6 \%)^{3}$ and the high diagnostic yield (approximately $90 \%-95 \%),{ }^{1,2,4}$ stereotactic biopsies of supratentorial lesions demonstrate a good risk-benefit ratio for acquiring diagnostic confidence before deciding on a definite therapy. On the other hand, there has been a long-lasting controversial debate around brainstem lesions (i.e., midbrain, pons, and medulla) considering the risk-benefit ratio of stereotactic tissue sampling. ${ }^{5-11}$ First, because of the high density of eloquent tissue along the trajectory and within the sampling area, brainstem biopsies have been considered to bear a higher risk for transient and permanent perioperative morbidity (ranging between $0 \%$ and 27.8\%). ${ }^{12}$ Second, the benefit is questioned as brainstem lesions are usually well described by radiological means $s^{5,6,13}$ and therapeutic options are often limited. The fact that brainstem lesions are predominantly found in pediatric patients further perpetuates the controversy. ${ }^{14}$ However, two major

ABBREVIATIONS BFR = bone fiducial registration; LSR = laser-based surface registration; SPT = surgical procedure time; STS = skin-to-skin; TCA = transcerebellar approach; TFA = transfrontal approach. 
developments are currently reigniting the debate. 1) Modern neurooncological therapies are highly dependent on molecular and genetic classification necessitating tissue sampling..$^{15}$ 2) The introduction of robotic technology facilitates new trajectories to the brainstem (e.g., transcerebellar). ${ }^{16-19}$

In general, stereotactic neurosurgeons gain access to the brainstem via a transfrontal approach (TFA) or a transcerebellar approach (TCA) through the cerebral or cerebellar peduncles. The TFA to the brainstem is characterized by one of the longest trajectories in stereotactic neurosurgery and a narrow surgical corridor affecting numerous highly eloquent regions (e.g., internal capsule, basal ganglia, and cerebral crura). ${ }^{12,20}$ In contrast, the TCA involves a shorter trajectory length and a wider stereotactic corridor. Nevertheless, the TCA is considered more complex, time consuming, and risky due to the modified patient and inverted frame positioning. ${ }^{21,22}$ As a result of these drawbacks, the TFA is still favored in conventional frame-based stereotaxy. ${ }^{12,20}$ In contrast, robot-assisted stereotaxy potentially dissolves the disadvantages of the TCA by the omission of the stereotactic frame. In this regard, robotic stereotaxy might improve the risk-benefit ratio of brainstem biopsies. However, studies on robot-assisted brainstem biopsies are rare and include only small (pediatric) patient cohorts (with < 20 cases). ${ }^{16-19,23-25}$ In addition, complication rates of robot-assisted brainstem biopsies differ considerably. ${ }^{16-18,23}$

Therefore, the aim of this single-center cohort study was to evaluate the risk-benefit ratio of brainstem biopsies using 2 different framed and nonframed neurosurgical robots. In addition to standard quality indicators (e.g., skin-to-skin [STS] time, diagnostic yield, and complication rates), a special focus is set on trajectory selection for reducing surgical morbidity.

\section{Methods \\ Patient Inclusion}

This retrospective study included 25 patients $(45.0 \pm$ 26.4 years [range 5-80 years], 7 female) who underwent 26 robot-assisted (Renaissance, Mazor Robotics or ROSA One, Zimmer Biomet Robotics) brainstem biopsies via a TCA. The inclusion criteria were 1) a lesion affecting the brainstem or cerebellar peduncle and 2) the presence of postoperative CT or MRI. One patient underwent biopsy twice. The study was approved by the local ethics committee and was performed in accordance with the Declaration of Helsinki. Patient characteristics and surgical procedures are summarized in Table 1.

\section{Robotic Systems and Procedural Workflow}

Robot-assisted, transcerebellar biopsies were performed with the assistance of 2 robotic systems, which have been previously described in detail. ${ }^{26-28}$ The Renaissance robot (Mazor Robotics; robot 1) is a framed, supervisory-controlled robot. The frame fixed on the cranial bone interfaces the robotic device with the patient's head (Fig. 1B). After positioning the robotic arm according to preplanned, image-guided trajectories, the surgeon directs the biopsy needle to the target. The ROSA One (Zimmer
Biomet; robot 2) is a serial robot with six degrees of freedom (Fig. 1A) ${ }^{29}$ It can be used in a framed and nonframed mode ${ }^{28-31}$ and has been successfully used for intracranial biopsies. ${ }^{27,32}$ ROSA-assisted, transcerebellar biopsies were performed using a CT-based bone fiducial registration (BFR; WayPoint, FHC Inc.).

Trajectories were planned using the Renaissance or ROSA One software. Entry and target points were defined on a high-resolution, T1-weighted, contrast-enhanced MPRAGE sequence (slice thickness $1.0 \mathrm{~mm}$, TR 3200 msec, TE $381 \mathrm{msec}$, TI $1100 \mathrm{msec}$, flip angle $8^{\circ}$, pixel bandwidth 130; pixel spacing $1 / 1 \mathrm{~mm}$; matrix $256 \times 256$ ) and, where required, on additional high-resolution, T2SPC-weighted MR sequences (slice thickness $1.0 \mathrm{~mm}$, TR $3200 \mathrm{msec}$, TE $408 \mathrm{msec}$, flip angle $120^{\circ}$, pixel bandwidth 750 , pixel spacing $0.976 / 0.976 \mathrm{~mm}$, matrix $256 \times$ 256) or FLAIR sequences. After placing the bone fiducials at bedside (robot 2) or the Renaissance frame on the day of surgery, an additional preoperative $0.5 \mathrm{~mm}$ to 1.0 mm contrast-enhanced CT scan (Siemens Healthineers) was performed. The MR sequences were merged with the CT scan and the trajectories were verified. After positioning the patient supine, with their head rotated to the contralesional side, and performing the robot-to-patient registration, subsequent intraoperative steps were identical for both robotic systems. Following indication of the entry point by the stereotactic device, the patient's hair was shaved locally. The skin was incised and neck muscles dissected. A burr hole was performed using a $14-\mathrm{mm}$ drill bit. The dura mater was coagulated and incised. Finally, the biopsy needle $(1.8 \mathrm{~mm} \times 250 \mathrm{~mm}$, BrainPro, Pajunk) was introduced and 4 to 6 core samples were taken at the target point and, optionally, $5 \mathrm{~mm}$ posterior to the target. A frozen section examination was performed, and results were awaited to confirm diagnostic samples before the surgery was terminated.

To exclude surgery-related complications, a CT or MR scan was obtained in all patients within 24 hours after robot-assisted biopsy. Morbidity and mortality were systematically analyzed, as well as the histological results, STS time, and surgical procedure time (SPT). The SPT was defined as the surgeon's entry and exit to and from the operating room, and included the positioning of the patient, the intraoperative registration procedure, asepsis, and the STS time. ${ }^{27}$

\section{Trajectory Characteristics}

MATLAB (R2019a, The Mathworks Inc.), SPM 12 (https://www.fil.ion.ucl.ac.uk/spm/software/spm12/), and the Lead-DBS toolbox (https://www.lead-dbs.org) were used for systematic evaluation and visualization of the position and length of transcerebellar trajectories. ROSA and MAZOR header files were exported from the robotic software, and the entry and target points of trajectories were delineated into the preoperative MRI using custom-based MATLAB scripts. These images were processed using the Lead-DBS software. The general concept of the LeadDBS toolbox has been described elsewhere. ${ }^{33}$ The advantage of Lead-DBS is the possibility of showing trajectories of different patients in a common space. In summary, preand postoperative MR or CT scans were coregistered lin- 
TABLE 1. Patient characteristics

\begin{tabular}{|c|c|c|c|c|}
\hline & Overall $(n=25)$ & Pediatric Pts $(n=7)$ & Adult Pts $(n=18)$ & $p$ Value $^{*}$ \\
\hline Mean age \pm SD (range), yrs & $45.0 \pm 26.4(5-80)$ & $11.0 \pm 4.3(5-16)$ & $58.2 \pm 17.9(20-80)$ & $<0.001$ \\
\hline Sex, F:M & $7: 18$ & $1: 6$ & $6: 12$ & 0.626 \\
\hline \multicolumn{5}{|l|}{ Symptoms at presentation } \\
\hline Ataxia/uncertain gait & $18 / 25(72.0)$ & $7 / 7(100)$ & $11 / 18(61.1)$ & 0.133 \\
\hline Dysarthria & $7 / 25(28.0)$ & $3 / 7(42.9)$ & $4 / 18(22.2)$ & 0.355 \\
\hline Nystagmus & $2 / 25(8.0)$ & $1 / 7(14.3)$ & $1 / 18(5.6)$ & 0.490 \\
\hline Cranial nerve disorder & $16 / 25(64.0)$ & $5 / 7(71.4)$ & $11 / 18(61.1)$ & $>0.99$ \\
\hline Nausea/emesis & $5 / 25(20.0)$ & $1 / 7(14.3)$ & $4 / 18(22.2)$ & $>0.99$ \\
\hline (Hemi-)paresis & $9 / 25(36.0)$ & $2 / 7(28.6)$ & $7 / 18(38.9)$ & $>0.99$ \\
\hline Hypesthesia & $4 / 25(16.0)$ & $0 / 7(0)$ & $4 / 18(22.2)$ & $>0.99$ \\
\hline Headache & $3 / 25(12.0)$ & $1 / 7(14.3)$ & $2 / 18(11.1)$ & $>0.99$ \\
\hline Cognitive deficits/lethargy & $2 / 25(8.0)$ & $1 / 7(14.3)$ & $1 / 18(5.6)$ & 0.490 \\
\hline \multicolumn{5}{|l|}{ Robotic system } \\
\hline ROSA One & $21 / 26(80.8)$ & $6 / 7(85.7)$ & $15 / 19(78.9)$ & $>0.99$ \\
\hline Renaissance & $5 / 26(19.2)$ & $1 / 7(14.3)$ & $4 / 19(21.1)$ & $>0.99$ \\
\hline Mean SPT $\pm S D$, mins & $91.8 \pm 27.1$ & $80.4 \pm 12.7$ & $96.0 \pm 29.9$ & 0.355 \\
\hline Mean STS time \pm SD, mins & $49.5 \pm 23.7$ & $41.7 \pm 12.6$ & $52.3 \pm 26.4$ & 0.311 \\
\hline \multicolumn{5}{|l|}{ Complications } \\
\hline Overall & $2 / 26(7.7)$ & $1 / 7(14.3)$ & $1 / 19(5.3)$ & 0.474 \\
\hline Transient neurological deficits & $1 / 26(3.8)$ & $1 / 7(14.3)$ & $0 / 19(0)$ & 0.269 \\
\hline Permanent neurological deficits & $0 / 26(0)$ & $0 / 7(0)$ & $0 / 19(0)$ & $>0.99$ \\
\hline Diagnostic yield & $25 / 26(96.2)$ & $7 / 7(100)$ & $18 / 19(94.7)$ & $>0.99$ \\
\hline \multicolumn{5}{|l|}{ Histology } \\
\hline Glioma & $14 / 25(56)$ & $6 / 7(85.7)$ & $8 / 18(44.4)$ & 0.090 \\
\hline Medulloblastoma & $1 / 25(4)$ & $1 / 7(14.3)$ & $0 / 18(0)$ & 0.280 \\
\hline Lymphoma & $6 / 25(24)$ & $0 / 7(0)$ & 6/18 (33.3) & 0.137 \\
\hline Inflammation & $4 / 25(16)$ & $0 / 7(0)$ & $4 / 18(22.2)$ & 0.295 \\
\hline
\end{tabular}

early using SPM 12 and then spatially normalized into the MNI_ICBM_2009b_NLIN_ASYM space. ${ }^{33}$ Entry and target points of trajectories were manually localized due to the previous delineated points. Subsequently, transcerebellar trajectories were visualized in 3D space. The Cerebellum-SUIT atlas by Diedrichsen ${ }^{34}$ was used to evaluate anatomical localization (i.e., cerebellar lobule) of the entry points (Fig. 2A-E).

In all patients, we designed hypothetical transfrontal trajectories according to general stereotactic principles to compare these with the TCA (Fig. 3A and B). The trajectories were assessed for length and anatomical location according to the procedure described for the trajectories. The Brainstem Connectome Atlas ${ }^{35}$ and the MNI PD25 Subcortical Atlas $^{36}$ were used for graphical visualization of the basal ganglia and the midline pathways (Fig. 3C and D).

\section{Statistical Analysis}

All statistical tests were performed using MATLAB and IBM SPSS Statistics version 22.0 (IBM Corp.). Preoperative symptoms and histological results were compared between adult and pediatric patients using the chi-square and Fisher's exact tests. Differences in SPT, STS, and trajectory characteristics between robots as well as between pediatric and adult patients were evaluated with the Kruskal-Wallis test. The length of electrodes and target point coordinates in the TCA and TFA were compared using a paired t-test. Data are shown as mean $\pm \mathrm{SD}$. Any $\mathrm{p}$ values $<0.05$ were considered significant.

\section{Results}

\section{Clinical and Histological Characteristics}

Overall, 25 patients with a lesion within the brainstem underwent 26 robot-assisted surgeries (Table 1); 7 patients $(28.0 \%)$ were children ( $\leq 18$ years of age). The main symptoms at presentation were ataxia in 18 of 25 patients (72.0\%) and cranial nerve disorders in 16 patients (64.0\%). There were no significant differences in the preoperative 

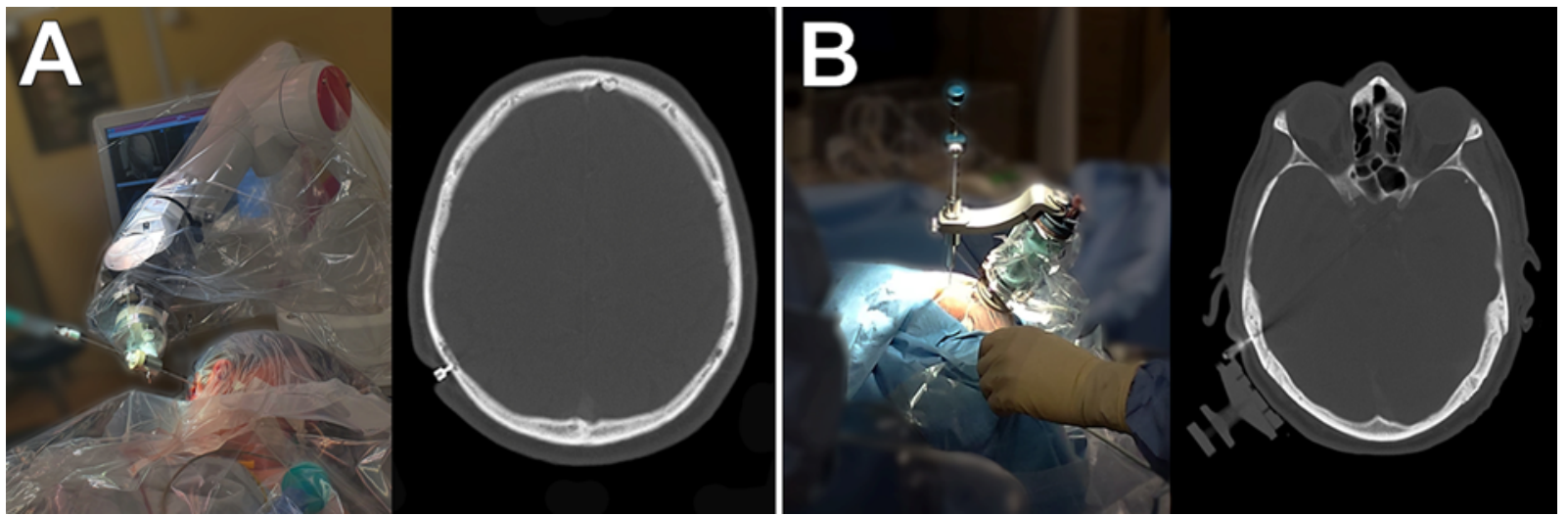

FIG. 1. Robots and registration techniques. A: The ROSA One robot in action assisting a brainstem biopsy (left). Registration is based on bone fiducials, as shown on the preoperative CT scan (right). B: The Renaissance robot in action (left). This robot registration is based on a frame mounted to the skull and a preoperative CT scan (right).

symptomology between pediatric and adult patients (Table 1). Biopsy was completed under robotic assistance in all cases. The mean SPT was $91.8 \pm 27.1$ minutes (range $60-176$ minutes) with a mean STS time of $49.5 \pm 23.7$ minutes (range 27-146 minutes). The SPT and STS time did not differ between pediatric and adult patients $(\mathrm{H}=$ $0.86, \mathrm{p}=0.355$ and $\mathrm{H}=1.03, \mathrm{p}=0.311$; Kruskal-Wallis).

In 25 of the $26(96.2 \%)$ biopsies, the procedure yielded a definite histopathological diagnosis. Gliomas (14/25, $56.0 \%)$ and lymphomas $(6 / 25,24.0 \%)$ represented the most frequent pathologies. The histological result was inconclusive in 1 patient, who received high-dose corticosteroids for 3 days prior to surgery. Inflammatory demyelinating lesions were found, which were deemed compatible with a corticosteroid-mitigated lymphoma. After repeating the robot-assisted biopsy 9 days later, final histological results revealed a lymphoma. Lymphoma-specific therapy was initiated, which improved the patient's clinical condition significantly. All pediatric patients had intrinsic primary brain tumors, while 10 of the $18(55.5 \%)$ adult patients presented other causes $\left(\chi^{2}=6.48, p=0.020\right.$; Fisher's exact test).

\section{Safety of Robot-Assisted Transcerebellar Biopsies}

There was no surgery-related mortality or permanent neurological deficit within the presented patient cohort. Of the 26 biopsies, 24 (92.3\%) were performed without complications. Only 2 cases (7.7\%) involved a transient morbidity after surgery. The postoperative CT scan of 1 patient, who underwent surgery with the assistance of robot 1 , showed a small epidural hematoma associated with the fixation screws of the robot. However, the patient had no new neurological symptoms, and surgical revision was not necessary as the hematoma regressed spontaneously. During a brainstem biopsy assisted by robot 2 , the patient exhibited a brief intraoperative asystole. Cardiac rhythm and blood pressure normalized within seconds after a short interruption of surgery without further intervention. Post-
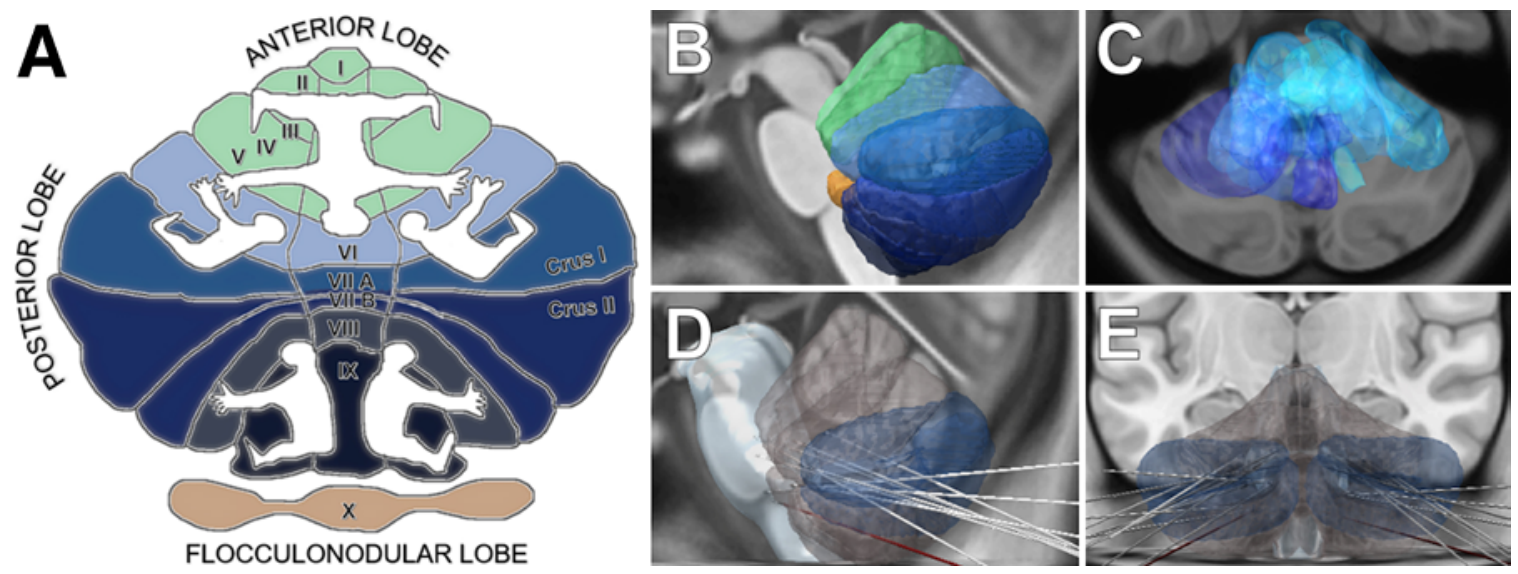

FIG. 2. Brainstem and cerebellar trajectories applying a TCA. A and B: Illustrations of the cerebellar lobes (green = anterior lobe, blue $=$ posterior lobe , and beige $=$ flocculonodular lobe) and lobules (I-X). C-E: Tumor localization (C), and lateral (D) and posterior $(E)$ visualization of trajectories (blue indicates crus I and II of the cerebellum and red trajectories demonstrate routes with an entry point through the lobule VIIb). 
operatively, 1 patient with preexisting facial paresis experienced transient worsening of symptoms. The CT scan revealed a minor intratumoral hemorrhage at the biopsy site. The patient completely recovered to the preoperative status within 3 days. Thus, only 1 (3.8\%) of 26 biopsies involved a transient neurological deterioration.

\section{Differences Between the Applied Robotic Systems}

The present study used 2 different framed and nonframed neurosurgical robots. While there were no significant differences in SPT $(87.2 \pm 9.1$ minutes and 92.9 \pm 29.9 minutes) between the robotic devices $(\mathrm{H}=0.01, \mathrm{p}$ $=0.922$; Kruskal-Wallis), robot 1 exerted a significantly longer STS time than robot $2(59.8 \pm 9.0$ minutes and 47.0 \pm 25.6 minutes, $\mathrm{H}=5.66, \mathrm{p}=0.017$; Kruskal-Wallis).

\section{Trajectory Characteristics}

Transcerebellar trajectories had a mean length of 48.4 $\pm 7.3 \mathrm{~mm}$ (range $36.6-61.3 \mathrm{~mm}$ ) and were localized on the right side in 14 of 26 cases (53.8\%). The trajectory length did not differ between pediatric and adult patients (49.4 \pm $6.4 \mathrm{~mm}$ [range $40.7-59.0 \mathrm{~mm}$ ] and $48.0 \pm 7.7 \mathrm{~mm}$ [range 36.6-61.3 mm], $\mathrm{H}=0.24, \mathrm{p}=0.623$; Kruskal-Wallis) or between the robotic systems $(53.3 \pm 8.1 \mathrm{~mm}$ [range 40.6$61.1 \mathrm{~mm}$ ] and $47.2 \pm 6.8 \mathrm{~mm}$ [range 36.6-61.3 mm], $\mathrm{H}=$ $2.75, p=0.970$; Kruskal-Wallis). Anatomically, biopsy was performed through crus I $(9 / 26,34.6 \%)$ or crus II $(15 / 26$, $57.7 \%)$ of the cerebellum in 24 of $26(92.3 \%)$ cases. In 2 cases $(7.7 \%)$ among the 26 biopsies, the entry point was located in lobule VIIb. Trajectories affected the dentate nucleus in 4 cases (15.4\%), with median localized lesions. Fastigial and interposed nuclei were avoided in all cases. Preferably, biopsy was performed in the posterior border of lesions within the cerebellar peduncles instead of the brainstem, avoiding any conflict with the midline pathways and cranial nerve nuclei (Fig. 3D). The patient who experienced a brief intraoperative asystole underwent a medial biopsy, probably affecting the trigeminal nucleus.

For comparison, we retrospectively reconstructed transfrontal trajectories to the brainstem lesions. In TFA reconstructions, lesions were attained through a contralateral entry point in 21 of 26 cases (80.8\%) (Fig. 3A and B), and trajectories were significantly longer $(107.4 \pm 11.8 \mathrm{~mm}$ [range 77.7-129.1 mm]) compared with TCA trajectories $(\mathrm{t}=-20.09, \mathrm{p}<0.001$; paired $\mathrm{t}$-test). Anatomically, transfrontal trajectories regularly crossed the internal capsule, basal ganglia (i.e., caudate nucleus, putamen, and globus pallidus), thalamus, and cerebral peduncle (Fig. 3C). Due to the narrow anatomical corridor of the TFA, target points were significantly more medial and anterior in comparison with the TCA trajectories (x-direction: $-3.4 \pm 7.2 \mathrm{~mm}$, $\mathrm{t}=-2.17, \mathrm{p}=0.042 ; \mathrm{y}$-direction: $-3.9 \pm 8.4 \mathrm{~mm}, \mathrm{t}=2.11, \mathrm{p}$ $=0.048$; paired t-test). Z-coordinates did not significantly differ between TCA and TFA target points $(1.0 \pm 7.2 \mathrm{~mm}$, $\mathrm{t}=0.64, \mathrm{p}=0.530$; paired t-test) (Fig. 4).

\section{Discussion}

The present study represents one of the largest series of robot-assisted, transcerebellar biopsies. Our study demonstrates a high diagnostic yield (i.e., 96.2\%) while exerting

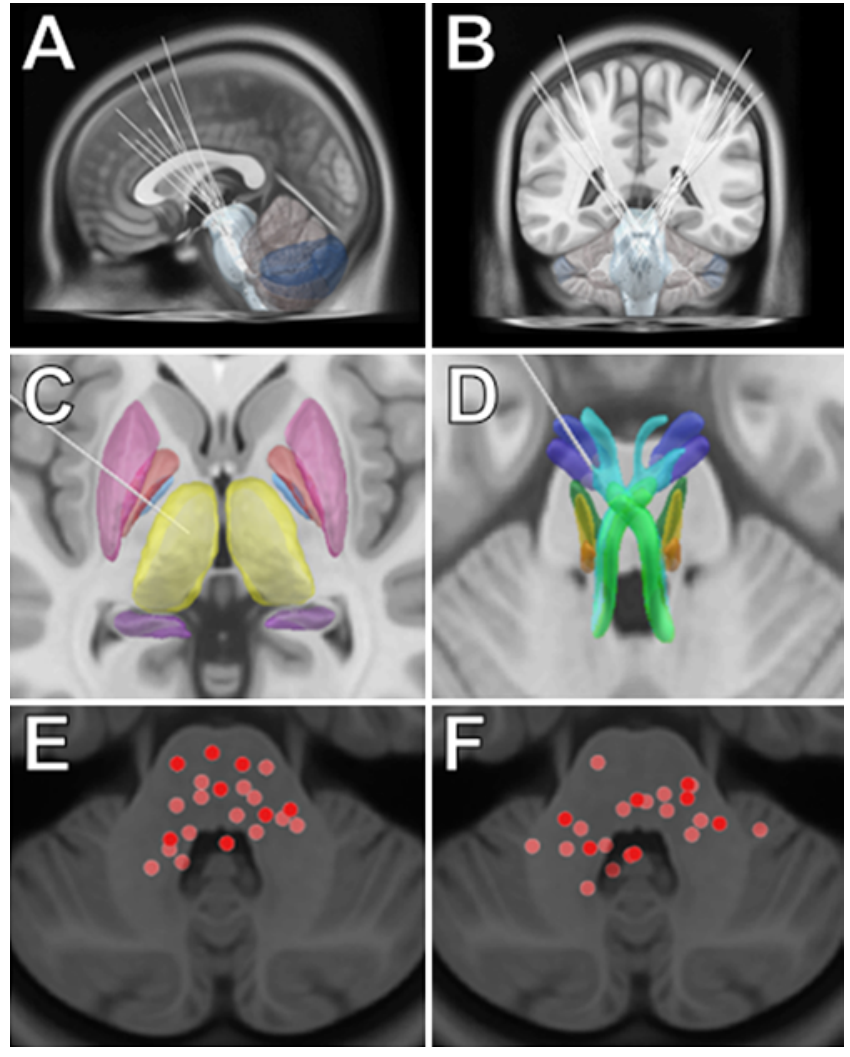

FIG. 3. Trajectories to the brainstem applying a TFA. A and B: Lateral (A) and anterior $(\mathrm{B})$ views (blue $=$ crus I and II of the cerebellum). C: Illustration showing an exemplary trajectory affecting the thalamus (light yellow $=$ thalami, dark blue = internal globus pallidus, red = external globus pallidus, pink = putamen, and purple = hippocampus). D: Illustration demonstrating functional tracts at the level of the pons (dark blue $=$ corticospinal and frontopontine tract, dark green $=$ medial lemniscus, orange = lateral lemniscus, yellow = spinothalamic tract, lime green = cerebellorubral tract, and light blue $=$ anterior spinocerebellar tract). $\mathrm{E}$ and $\mathrm{F}$ : Illustrations demonstrating the target points (red) of the TFA (E) and TCA (F).

no surgical mortality and no permanent morbidity. Only $3.8 \%$ of the cases involved a transient neurological deterioration. This is in good accordance with the diagnostic yield (approximately 95\%), ${ }^{12,16-19,23-25}$ as well as transient (approximately 7.8\%) and permanent (approximately $1.7 \%$ ) morbidity described in frame-based supratentorial biopsies. $^{12}$

The risk-benefit ratio of stereotactic biopsies of brainstem lesions has been discussed excessively in pediatric patients..$^{5-9,13}$ Since lesion classification is often ensured by typical neuroradiological features alone and therapeutic options are limited, $, 5,6,13$ the risk-benefit ratio of stereotactic brainstem biopsies is often questioned. Hence, a calculated therapy (i.e., radiotherapy) is introduced to avoid surgical morbidity. ${ }^{5,6,13}$ The present study is one of few studies investigating brainstem biopsies in an adult patient cohort. ${ }^{10,37}$ Notably, the histological findings differ significantly from those of pediatric patients. A remarkably high portion of adult patients show other histological findings (e.g., inflammation and lymphomas), which are accessible to specific treatment regimens (e.g., chemotherapy) 

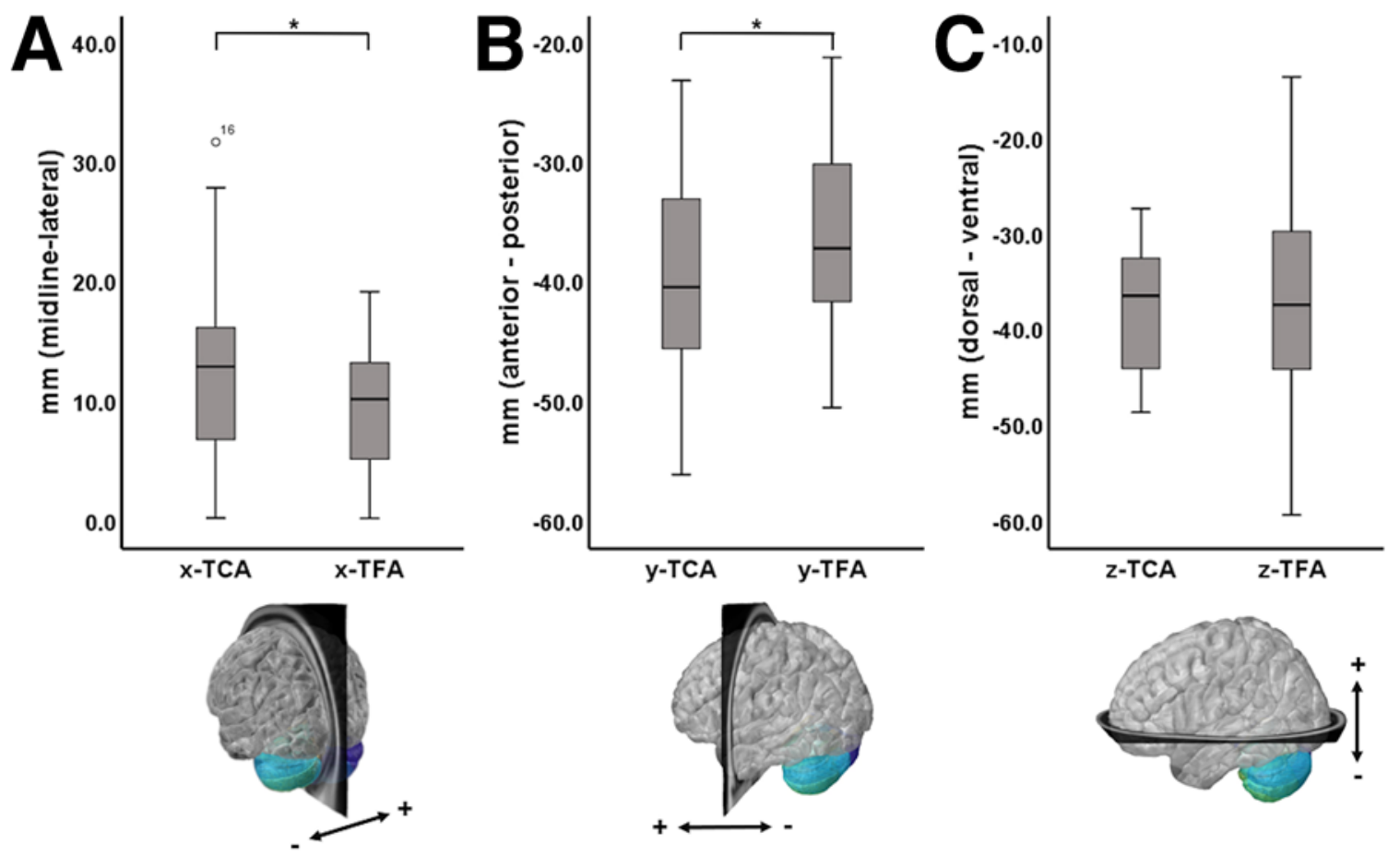

FIG. 4. Target point coordinates of TCA and TFA trajectories. A and B: Boxplots showing significant differences of $x$ - and $y$ coordinates between approaches (x-direction: $-3.41 \mathrm{~mm}$; $y$-direction: $-3.9 \mathrm{~mm}$ ). The $\mathrm{x}$-coordinates are normalized to one side. C: Boxplot showing that the z-coordinates did not significantly differ between TCA and TFA target points. ${ }^{*} p<0.05$; paired t-test.

necessitating previous tissue sampling. ${ }^{10,12,37}$ Furthermore, the increasing adaption of treatment strategies based on molecular tissue characteristics (i.e., targeted therapy) has recently reignited the debate about the risk-benefit ratio of brainstem biopsies in pediatric patients. We hypothesized that the introduction of robotic technology has the potential to turn the tide by improving accessibility in surgical (i.e., transcerebellar) approaches to the brainstem.

In conventional frame-based stereotaxy, lesions of the brainstem have been targeted less frequently by transcerebellar than transfrontal trajectories. ${ }^{12}$ The TCA is considered more complex because of the necessity of a prone or semisitting position and a modified attachment of the stereotactic ring, respectively. ${ }^{21,38}$ Robotic technology certainly enables a simplified application of the TCA, avoiding these restrictions as well as the disadvantages of the TFA (i.e., higher inaccuracy due to long trajectories, a small surgical corridor, and extensive effects on eloquent structures). In our opinion, the advantages of the TCA are a shorter trajectory length and a larger anatomical corridor, enabling the avoidance of eloquent tissue.

The cerebellum is involved in several sensorimotor, associative, and cognitive functions..$^{39,40}$ While the frontal cerebellar lobe as well as lobule VIII are primarily involved in sensorimotor functions, the main parts of the posterior lobe are associated with cognitive and limbic functions. The cerebellar nuclei are located medially and are responsible for the planning, initiation, and regulation of movements (dorsal region of the dentate nucleus, interposed nucleus) as well as nonmotor functions like consciousness and emotion control (ventral region of the dentate nucleus, fastigial nucleus). ${ }^{39,40}$ The present study demonstrates that positioning cerebellar trajectories through crus I or II and the middle cerebellar peduncle enables a secure biopsy avoiding sensitive anatomical areas. This approach provides a comparatively wide stereotactic corridor increasing the accessibility of the brainstem for biopsies in all directions. Thus, we prefer tissue sampling in dorsal tumor parts instead of within the brainstem itself, avoiding the midline pathways and the (motor) nuclei of the cranial nerves. Our study indicates that these areas are hardly accessible with the TFA.

The present study had no surgical mortality and transient morbidity (i.e., 1 patient with epidural bleeding and 1 patient with a brief intraoperative asystole and transient, postoperative facial weakness). These results oppose previous studies that demonstrated an overall morbidity of up to $28.6 \%$ and a permanent morbidity of up to $14.3 \%$ in robot-assisted brainstem biopsies. ${ }^{16-19,23-25}$ These discrepancies may be caused by the small sample sizes of the available studies and different definitions of morbidity. For example, some studies consider intraoperative bradycardia/asystole as a complication..$^{16}$ These events are, however, well explained by the trigeminocardiac reflex, ${ }^{41}$ which can occur when the trigeminal sensory nucleus is affected and usually does not pose a significant risk to the patient if the surgical procedure is paused. In our study, we experienced an intraoperative asystole in a single case. Notably, this patient had a rather medial target point. In contrast, permanent disorders such as hemiparesis and permanent cranial nerve disorders can significantly impair a patient's quality of life. Previous studies suggest a higher rate of permanent 
deficits with the TFA compared with the TCA.16-19,23-25 We hypothesize that widening of the stereotactic corridor by the TCA might help to prevent the occurrence of such events by distancing the trajectory from delicate areas. However, due to the eloquent region, some cases of permanent morbidity will not be completely avoidable in larger series. Profound registration and anatomical-functional trajectory selection (as mentioned above) are therefore essential to reduce mortality and morbidity.

However, not only anatomical aspects but also technical details contribute to the results and should be considered in the calculation of the risk-benefit ratio in robot-assisted brainstem biopsies. Studies resulting in a high complication rate during robot-assisted biopsies of the brainstem mainly used laser-based surface registration (LSR). ${ }^{16,23}$ Previous studies, however, demonstrated a higher inaccuracy of LSR compared with frame-based registration and BFR, especially in long trajectories and trajectories with entry points distant to the registration plane (i.e., the face). ${ }^{27,31,32,42}$ In our opinion, LSR should be avoided for brainstem biopsies in both the TFA and TCA. In the present study, we have mainly applied BFR, which has been shown to increase the accuracy of robot-assisted stereotaxy to the level of conventional frame-based techniques. ${ }^{26}$ In contrast, several groups apply stereotactic robots in a frame-based configuration. ${ }^{17}$ While increasing the accuracy, this approach entails the same disadvantages (e.g., patient positioning and frame fixation) as conventional frame-based stereotaxy. Notably, one of the robots used in this study is classified as a framed robot since it uses a head-mounted frame..$^{28}$ Due to its small size, this frame did not impede patient positioning and trajectory planning, which, thus, blurs the boundaries between frame-based and frameless robotics. ${ }^{43}$

In summary, the robot-assisted TCA technique is highly suitable for improving surgical accessibility to the brainstem. As survival in patients with brainstem gliomas has remained static and diffuse pontine gliomas are still a main cause of death in children with brain tumors, ${ }^{44}$ the implementation of robot-assisted transcerebellar brainstem biopsies in a routine manner could help in the understanding of genetic patterns and biological correlation to drug activity. ${ }^{44,45}$ Registry-based studies on this rare but deadly tumor entity, which are actually not feasible due to the missing histological tissue sampling, may become possible. Finally, in adult patients, who often show other histological findings (e.g., inflammation and lymphomas), tissue sampling may be decisive for the choice of therapy. Acceptance of routine and safe stereotactic biopsy can, therefore, have a massive impact on improving management strategies and introducing new ones.

\section{Limitations}

The present study represents a retrospective analysis of robot-assisted brainstem biopsies using a TCA. The lack of prospective randomized studies, small patient cohorts, and technical differences between robotic studies impedes a conclusion on the differences between the TFA and TCA. Furthermore, the potential for new-onset, subtle cognitive deficits postoperatively has to be discussed when undertaking a transcerebellar biopsy through crus I and II. Routine bedside examination and retrospective analysis may have missed these changes. In the future, prospective analyses should exert evaluation tests (e.g., the Schmahmann syndrome scale). However, limited cognitive deficits might be deemed acceptable in order to enable target-based therapy.

\section{Conclusions}

Brainstem biopsies remain a challenging procedure in stereotactic neurosurgery. From an anatomical-functional point of view, the TCA to the brainstem is advantageous compared with the TFA. In conventional frame-based stereotaxy, however, the realization of the TCA is impeded by the geometry and functionality of the available frames. Robot-assisted stereotaxy simplifies and increases the safety of brainstem biopsies, if appropriate registration and trajectory selection is applied. This technological advancement may potentially improve the risk-benefit ratio of brainstem biopsies in adult as well as in pediatric patients.

\section{References}

1. Apuzzo MLJ, Chandrasoma PT, Cohen D, Zee CS, Zelman V. Computed imaging stereotaxy: experience and perspective related to 500 procedures applied to brain masses. Neurosurgery. 1987;20(6):930-937.

2. Sawin PD, Hitchon PW, Follett KA, Torner JC. Computed imaging-assisted stereotactic brain biopsy: a risk analysis of 225 consecutive cases. Surg Neurol. 1998;49(6):640-649.

3. Grossman R, Sadetzki S, Spiegelmann R, Ram Z. Haemorrhagic complications and the incidence of asymptomatic bleeding associated with stereotactic brain biopsies. Acta Neurochir (Wien). 2005;147(6):627-631.

4. Dammers R, Haitsma IK, Schouten JW, Kros JM, Avezaat CJ, Vincent AJ. Safety and efficacy of frameless and framebased intracranial biopsy techniques. Acta Neurochir (Wien). 2008;150(1):23-29.

5. Albright AL, Packer RJ, Zimmerman R, Rorke LB, Boyett J, Hammond GD. Magnetic resonance scans should replace biopsies for the diagnosis of diffuse brain stem gliomas: a report from the Children's Cancer Group. Neurosurgery. 1993; 33(6):1026-1030.

6. Dellaretti M, Câmara BBA, Ferreira PHPB, da Silva Júnior JB, Arantes RME. Impact of histological diagnosis on the treatment of atypical brainstem lesions. Sci Rep. 2020;10(1): 11065.

7. Hankinson TC, Campagna EJ, Foreman NK, Handler MH. Interpretation of magnetic resonance images in diffuse intrinsic pontine glioma: a survey of pediatric neurosurgeons. $J$ Neurosurg Pediatr. 2011;8(1):97-102.

8. Kondziolka D, Lunsford LD. Results and expectations with image-integrated brainstem stereotactic biopsy. Surg Neurol. 1995;43(6):558-562.

9. Massager N, David P, Goldman S, et al. Combined magnetic resonance imaging- and positron emission tomography-guided stereotactic biopsy in brainstem mass lesions: diagnostic yield in a series of 30 patients. J Neurosurg. 2000;93(6):951957.

10. Rachinger W, Grau S, Holtmannspötter M, Herms J, Tonn JC, Kreth FW. Serial stereotactic biopsy of brainstem lesions in adults improves diagnostic accuracy compared with MRI only. J Neurol Neurosurg Psychiatry. 2009;80(10):1134-1139.

11. Rajshekhar V, Chandy MJ. Computerized tomography-guided stereotactic surgery for brainstem masses: a risk-benefit analysis in 71 patients. J Neurosurg. 1995;82(6):976-981.

12. Kickingereder P, Willeit P, Simon T, Ruge MI. Diagnostic 
value and safety of stereotactic biopsy for brainstem tumors: a systematic review and meta-analysis of 1480 cases. Neurosurgery. 2013;72(6):873-882.

13. Barkovich AJ, Krischer J, Kun LE, et al. Brain stem gliomas: a classification system based on magnetic resonance imaging. Pediatr Neurosurg. 1990-1991;16(2):73-83.

14. Merchant TE, Pollack IF, Loeffler JS. Brain tumors across the age spectrum: biology, therapy, and late effects. Semin Radiat Oncol. 2010;20(1):58-66.

15. Mader MMD, Rotermund R, Martens T, Westphal M, Matschke J, Abboud T. The role of frameless stereotactic biopsy in contemporary neuro-oncology: molecular specifications and diagnostic yield in biopsied glioma patients. $J$ Neurooncol. 2019;141(1):183-194.

16. Coca HA, Cebula H, Benmekhbi M, Chenard MP, Entz-Werle N, Proust F. Diffuse intrinsic pontine gliomas in children: interest of robotic frameless assisted biopsy. A technical note. Neurochirurgie. 2016;62(6):327-331.

17. Dawes W, Marcus HJ, Tisdall M, Aquilina K. Robot-assisted stereotactic brainstem biopsy in children: prospective cohort study. J Robot Surg. 2019;13(4):575-579.

18. Gupta M, Chan TM, Santiago-Dieppa DR, et al. Robot-assisted stereotactic biopsy of pediatric brainstem and thalamic lesions. J Neurosurg Pediatr. 2021;27(3):317-324.

19. Haegelen C, Touzet G, Reyns N, Maurage CA, Ayachi M, Blond S. Stereotactic robot-guided biopsies of brain stem lesions: experience with 15 cases. Neurochirurgie. 2010;56(5): 363-367.

20. Steck J, Friedman WA. Stereotactic biopsy of brainstem mass lesions. Surg Neurol. 1995;43(6):563-568.

21. Abernathey CD, Camacho A, Kelly PJ. Stereotaxic suboccipital transcerebellar biopsy of pontine mass lesions. J Neurosurg. 1989;70(2):195-200.

22. Horisawa S, Nakano H, Kawamata T, Taira T. Novel use of the Leksell gamma frame for stereotactic biopsy of posterior fossa lesions. World Neurosurg. 2017;107:1-5.

23. Carai A, Mastronuzzi A, De Benedictis A, et al. Robot-assisted stereotactic biopsy of diffuse intrinsic pontine glioma: a single-center experience. World Neurosurg. 2017;101:584588 .

24. Dellaretti M, Reyns N, Touzet G, et al. Stereotactic biopsy for brainstem tumors: comparison of transcerebellar with transfrontal approach. Stereotact Funct Neurosurg. 2012;90(2): 79-83.

25. De Benedictis A, Trezza A, Carai A, et al. Robot-assisted procedures in pediatric neurosurgery. Neurosurg Focus. 2017;42(5):E7.

26. Machetanz K, Grimm F, Wuttke TV, et al. Frame-based and robot-assisted insular stereoelectroencephalography via an anterior and posterior oblique approach. J Neurosurg. 2021; 135(5):1477-1486.

27. Machetanz K, Grimm F, Schuhmann M, Tatagiba M, Gharabaghi A, Naros G. Time efficiency in stereotactic robotassisted surgery: an appraisal of the surgical procedure and surgeon's learning curve. Stereotact Funct Neurosurg. 2021; 99(1):25-33.

28. Naros G, Machetanz K, Grimm F, Roser F, Gharabaghi A, Tatagiba M. Framed and non-framed robotics in neurosurgery: a 10-year single-center experience. Int J Med Robot. 2021;17(5):e2282.

29. Payne CJ, Dwyer G, Dimitrakakis E, Marcus HJ. Basic concepts in robotics. In: Neuromethods. Vol 162. Humana Press Inc.; 2021:3-34.

30. Smith JA, Jivraj J, Wong R, Yang V. 30 Years of neurosurgical robots: review and trends for manipulators and associated navigational systems. Ann Biomed Eng. 2016;44(4):836-846.

31. Machetanz K, Grimm F, Wang S, et al. Image-to-robot registration: the fate of robot-assisted stereotaxy. Int J Med Robot. 2021;17(5):e2288.
32. Lefranc M, Capel C, Pruvot-Occean AS, et al. Frameless robotic stereotactic biopsies: a consecutive series of 100 cases. J Neurosurg. 2015;122(2):342-352.

33. Horn A, Kühn AA. Lead-DBS: a toolbox for deep brain stimulation electrode localizations and visualizations. Neuroimage. 2015; 107:127-135.

34. Diedrichsen J. A spatially unbiased atlas template of the human cerebellum. Neuroimage. 2006;33(1):127-138.

35. Tang Y, Sun W, Toga AW, Ringman JM, Shi Y. A probabilistic atlas of human brainstem pathways based on connectome imaging data. Neuroimage. 2018;169:227-239.

36. Xiao Y, Fonov V, Chakravarty MM, et al. A dataset of multicontrast population-averaged brain MRI atlases of a Parkinson's disease cohort. Data Brief. 2017;12:370-379.

37. Manoj N, Arivazhagan A, Bhat DI, et al. Stereotactic biopsy of brainstem lesions: techniques, efficacy, safety, and disease variation between adults and children: a single institutional series and review. J Neurosci Rural Pract. 2014;5(1):32-39.

38. Jaradat A, Nowacki A, Fichtner J, Schlaeppi JA, Pollo C. Stereotactic biopsies of brainstem lesions: which approach? Acta Neurochir(Wien). 2021;163(7):1957-1964.

39. Grimaldi G, Manto M. Topography of cerebellar deficits in humans. Cerebellum. 2012;11(2):336-351.

40. Schmahmann JD. The cerebellum and cognition. Neurosci Lett. 2019;688:62-75.

41. Schaller B, Probst R, Strebel S, Gratzl O. Trigeminocardiac reflex during surgery in the cerebellopontine angle. J Neurosurg. 1999;90(2):215-220.

42. Spyrantis A, Cattani A, Woebbecke T, et al. Electrode placement accuracy in robot-assisted epilepsy surgery: a comparison of different referencing techniques including framebased CT versus facial laser scan based on CT or MRI. Epilepsy Behav. 2019;91:38-47.

43. Grimm F, Naros G, Gutenberg A, Keric N, Giese A, Gharabaghi A. Blurring the boundaries between frame-based and frameless stereotaxy: feasibility study for brain biopsies performed with the use of a head-mounted robot. J Neurosurg. 2015;123(3):737-742.

44. Hargrave D, Bartels U, Bouffet E. Diffuse brainstem glioma in children: critical review of clinical trials. Lancet Oncol. 2006;7(3):241-248.

45. Williams JR, Young CC, Vitanza NA, et al. Progress in diffuse intrinsic pontine glioma: advocating for stereotactic biopsy in the standard of care. Neurosurg Focus. 2020;48(1): E4.

\section{Disclosures}

The authors report no conflict of interest concerning the materials or methods used in this study or the findings specified in this paper.

\section{Author Contributions}

Conception and design: Naros, Machetanz. Acquisition of data: Naros, Machetanz, Grimm. Analysis and interpretation of data: Naros, Machetanz. Drafting the article: Machetanz, Naros. Critically revising the article: Naros, Grimm, Wang, Schuhmann, Tatagiba, Gharabaghi. Reviewed submitted version of manuscript: Naros. Approved the final version of the manuscript on behalf of all authors: Naros. Statistical analysis: Machetanz, Naros. Study supervision: Naros.

\section{Correspondence}

Georgios Naros: Eberhard Karls University, Tuebingen, Germany. georgios.naros@med.uni-tuebingen.de. 